\title{
Concise, Asymmetric Total Synthesis of Spirotryprostatin A
}

Tomoyuki Onishi, Paul R. Sebahar and Robert M. Williams*

Department of Chemistry, Colorado State University

Fort Collins, Colorado 80523

e-mail: rmw@chem.colostate.edu

\section{Supporting Information}

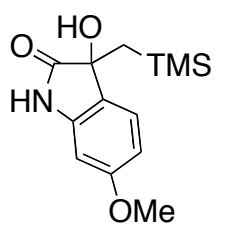

8

${ }^{1} \mathrm{H}$ NMR $\left(400 \mathrm{MHz}, \mathrm{CDCl}_{3}\right) \square \mathrm{CHCl}_{3}:-0.22(\mathrm{~s}, 9 \mathrm{H}), 1.49(\mathrm{~d}, \mathrm{~J}=10.2 \mathrm{~Hz}, 1 \mathrm{H}), 1.53(\mathrm{~d}, \mathrm{~J}=10.2 \mathrm{~Hz}, 1 \mathrm{H})$, $2.65(\mathrm{~s}, 1 \mathrm{H}), 3.79(\mathrm{~s}, 3 \mathrm{H}), 6.45(\mathrm{~d}, \mathrm{~J}=1.8 \mathrm{~Hz}, 1 \mathrm{H}), 6.56(\mathrm{dd}, \mathrm{J}=1.8,6.3 \mathrm{~Hz}, 1 \mathrm{H}), 7.23(\mathrm{~d}, \mathrm{~J}=6.3 \mathrm{~Hz}$, 1H), 7.88 (bs, $1 \mathrm{H}) ;{ }^{13} \mathrm{C} \mathrm{NMR}\left(100 \mathrm{MHz}, \mathrm{CDCl}_{3}\right) \square \mathrm{CHCl}_{3}:-1.1,28.3,55.5,75.5,97.5,107.5,123.4$, 125.4, 141.1, 161.2, 180.6; IR (NaCl/neat) 3388, 1714, 1634, 1507, 1351, 1251, 1151, 1125, $840 \mathrm{~cm}^{-}$ 1; HRMS (FAB+) calcd for $\mathrm{C}_{13} \mathrm{H}_{19} \mathrm{O}_{3} \mathrm{NSi}(\mathrm{m} / \mathrm{z}) 265.1134$, found $(\mathrm{m} / \mathrm{z}) 265.1132$.

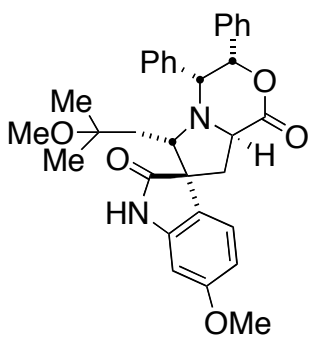

6

$[\square]_{D}^{24}=-46.0\left(\mathrm{CHCl}_{3}, \mathrm{c}=1\right) ;{ }^{1} \mathrm{H}$ NMR $\left(400 \mathrm{MHz}, \mathrm{CDCl}_{3}\right) \square \mathrm{CHCl}_{3}: 1.02(\mathrm{~s}, 3 \mathrm{H}), 1.09(\mathrm{~s}, 3 \mathrm{H}), 1.36(\mathrm{dd}$, $\mathrm{J}=5.6,15.6 \mathrm{~Hz}, 1 \mathrm{H}), 1.61(\mathrm{dd}, \mathrm{J}=2.1,15.6 \mathrm{~Hz}, 1 \mathrm{H}), 2.44(\mathrm{dd}, \mathrm{J}=8.0,12.6 \mathrm{~Hz}, 1 \mathrm{H}), 2.69$ (dd, J= 10.4, $12.6 \mathrm{~Hz}, 1 \mathrm{H}$ ), $3.04(\mathrm{~s}, 3 \mathrm{H}), 3.81(\mathrm{~s}, 3 \mathrm{H}), 4.01$ (dd, J=2.1, $5.6 \mathrm{~Hz}, 1 \mathrm{H}), 4.46(\mathrm{dd}, \mathrm{J}=8.0,10.4 \mathrm{~Hz}, 1 \mathrm{H}$ ), $4.85(\mathrm{~d}, \mathrm{~J}=2.6 \mathrm{~Hz}, 1 \mathrm{H}), 6.29(\mathrm{~d}, \mathrm{~J}=2.6 \mathrm{~Hz}, 1 \mathrm{H}), 6.55(\mathrm{~d}, \mathrm{~J}=2.1 \mathrm{~Hz}, 1 \mathrm{H}), 6.57(\mathrm{dd}, \mathrm{J}=2.1,8.4 \mathrm{~Hz}, 1 \mathrm{H})$, 6.99-7.05 (m, 2H), 7.13-7.33 (m, 9H), 8.35 (bs, $\left.1 \mathrm{H}) ;{ }^{13} \mathrm{C} \mathrm{NMR} \mathrm{(100} \mathrm{MHz,} \mathrm{CDCl}_{3}\right) \square \mathrm{CHCl}_{3}: 23.5,25.0$, $41.4,44.5,49.1,55.5,56.5,56.7,59.2$, 64.6, 73.3, 76.4, 97.3, 107.4, 122.6, 125.5, 125.7, 127.2, 127.6, 128.0, 128.3, 128.8, 136.8, 136.8, 141.6, 160.2, 172.2, 179.2; IR (NaCl/neat) 1726, 1631 , $1505,1271,1238,1193,1148,755,698 \mathrm{~cm}^{-1}$; HRMS (FAB+) calcd for $\mathrm{C}_{32} \mathrm{H}_{35} \mathrm{O}_{5} \mathrm{~N}_{2}(\mathrm{~m} / \mathrm{z}) 527.2546$, found $(\mathrm{m} / \mathrm{z}) 527.2540$. 


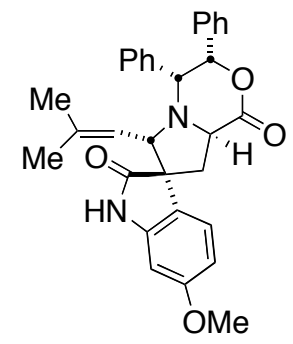

9

$[\square]_{D}^{24}=-36.4\left(\mathrm{CHCl}_{3}, \mathrm{c}=0.55\right) ;{ }^{1} \mathrm{H}$ NMR $\left(400 \mathrm{MHz}, \mathrm{CDCl}_{3}\right) \square \mathrm{CHCl}_{3}: 1.54(\mathrm{~s}, 3 \mathrm{H}), 1.74(\mathrm{~s}, 3 \mathrm{H}), 2.48$ (dd, J= 8.4, 13.0 Hz, 1H), $2.75(\mathrm{dd}, \mathrm{J}=9.6,13.0 \mathrm{~Hz}, 1 \mathrm{H}), 3.80$ (s, 3H), 4.27 (d, J= 3.0 Hz, 1H), 4.52 (d, $\mathrm{J}=9.4 \mathrm{~Hz}, 1 \mathrm{H}), 4.54(\mathrm{dd}, \mathrm{J}=8.4,9.6 \mathrm{~Hz}, 1 \mathrm{H}), 4.70(\mathrm{~d}, \mathrm{~J}=9.4 \mathrm{~Hz}, 1 \mathrm{H}), 6.18(\mathrm{~d}, \mathrm{~J}=3.0 \mathrm{~Hz}, 1 \mathrm{H}), 6.48(\mathrm{~d}$, $\mathrm{J}=2.3 \mathrm{~Hz}, 1 \mathrm{H}), 6.56(\mathrm{dd}, \mathrm{J}=2.3,8.4 \mathrm{~Hz}, 1 \mathrm{H}), 7.02-7.07(\mathrm{~m}, 2 \mathrm{H}), 7.15-7.33(\mathrm{~m}, 9 \mathrm{H}), 7.75(\mathrm{bs}, 1 \mathrm{H}) ;{ }^{13} \mathrm{C}$ NMR $\left(100 \mathrm{MHz}, \mathrm{CDCl}_{3}\right) \square \mathrm{CHCl}_{3}: 18.6,26.2,40.0,55.5,56.3,56.6,60.2,67.5,97.2,107.2,120.8$, $122.1,125.6,125.7,127.4,127.9,128.0,128.4,128.9,136.2,136.4,139.9,141.2,160.1,168.5$, 171.9, 178.3; IR (NaCl/neat) 3261, 1723, 1631, 1504, 1453, 1193, 1153, 755, $698 \mathrm{~cm}^{-1}$; HRMS $(\mathrm{FAB}+)$ calcd for $\mathrm{C}_{31} \mathrm{H}_{31} \mathrm{O}_{4} \mathrm{~N}_{2}(\mathrm{~m} / \mathrm{z}) 495.2284$, found $(\mathrm{m} / \mathrm{z}) 495.2267$.

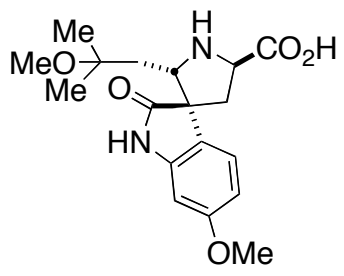

10

$[\square]_{D}^{23}=-18.4(\mathrm{MeOH}, \mathrm{c}=1) ;{ }^{1} \mathrm{H}$ NMR $\left(400 \mathrm{MHz}, \mathrm{CD}_{3} \mathrm{OD}\right) \square \mathrm{MeOH}: 1.17$ (s, 3H), 1.20 (s, 3H), 1.29 (dd, $\mathrm{J}=2.0,14.8 \mathrm{~Hz}, 1 \mathrm{H}), 1.72(\mathrm{dd}, \mathrm{J}=9.9,14.8 \mathrm{~Hz}, 1 \mathrm{H}), 2.49$ (dd, J=9.0, $13.1 \mathrm{~Hz}, 1 \mathrm{H}), 2.57$ (dd, J=9.0, $13.1 \mathrm{~Hz}, 1 \mathrm{H}), 3.24(\mathrm{~s}, 3 \mathrm{H}), 3.85(\mathrm{~s}, 3 \mathrm{H}), 4.19(\mathrm{dd}, \mathrm{J}=2.0,9.9 \mathrm{~Hz}, 1 \mathrm{H}), 4.51(\mathrm{t}, \mathrm{J}=9.0 \mathrm{~Hz}, 1 \mathrm{H}), 6.62(\mathrm{~d}$, $\mathrm{J}=2.0 \mathrm{~Hz}, 1 \mathrm{H}), 6.70(\mathrm{dd}, \mathrm{J}=2.0,8.4 \mathrm{~Hz}, 1 \mathrm{H}), 7.32(\mathrm{~d}, \mathrm{~J}=8.4 \mathrm{~Hz}, 1 \mathrm{H}) ;{ }^{13} \mathrm{C} N \mathrm{NMR}\left(100 \mathrm{MHz}, \mathrm{CD}_{3} \mathrm{OD}\right)$ $\mathrm{MeOH}: 22.2,25.0,40.8,42.6,49.6,56.0,59.1,61.3,63.7,75.1,98.9,108.4,121.3,125.8,144.2$, 162.5, 172.3, 178.6; IR (NaCl/neat) 2968, 1716, 1633, 1600, 1507, 1456, 1346, 1193, $1156 \mathrm{~cm}^{-1}$; HRMS (FAB+) calcd for $\mathrm{C}_{18} \mathrm{H}_{25} \mathrm{O}_{5} \mathrm{~N}_{2}(\mathrm{~m} / \mathrm{z}) 349.1764$, found $(\mathrm{m} / \mathrm{z}) 349.1778$. 


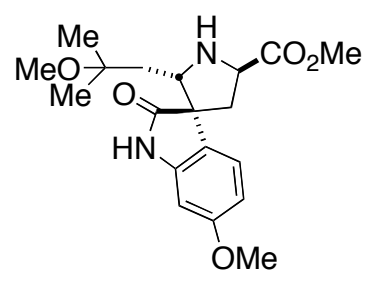

methyl ester of 10

$[\square]_{D}^{25}=-32.0\left(\mathrm{CHCl}_{3}, \mathrm{C}=1\right) ;{ }^{1} \mathrm{H} \mathrm{NMR}\left(400 \mathrm{MHz}, \mathrm{CDCl}_{3}\right) \square \mathrm{CHCl}_{3}: 0.96(\mathrm{dd}, \mathrm{J}=2.0,14.3 \mathrm{~Hz}, 1 \mathrm{H}), 1.05$ (s, 3H), 1.13 (s, 3H), 1.28 (dd, J= 9.7, $14.3 \mathrm{~Hz}, 1 \mathrm{H}$ ), 2.30 (dd, J= 9.0, $13.1 \mathrm{~Hz}, 1 \mathrm{H}$ ), 2.50 (dd, J= 6.8, $13.1 \mathrm{~Hz}, 1 \mathrm{H}), 3.11(\mathrm{~s}, 3 \mathrm{H}), 3.72(\mathrm{dd}, \mathrm{J}=2.0,9.7 \mathrm{~Hz}, 1 \mathrm{H}), 3.77(\mathrm{~s}, 3 \mathrm{H}), 3.80(\mathrm{~s}, 3 \mathrm{H}), 4.12$ (dd, J= 6.8, $9.0 \mathrm{~Hz}, 1 \mathrm{H}), 6.46(\mathrm{~d}, \mathrm{~J}=2.1 \mathrm{~Hz}, 1 \mathrm{H}), 6.54(\mathrm{dd}, \mathrm{J}=2.1,8.1 \mathrm{~Hz}, 1 \mathrm{H}), 7.32(\mathrm{~d}, \mathrm{~J}=8.1 \mathrm{~Hz}, 1 \mathrm{H}), 7.80(\mathrm{bs}$, $1 \mathrm{H}) ;{ }^{13} \mathrm{C} \mathrm{NMR}\left(100 \mathrm{MHz}, \mathrm{CDCl}_{3}\right) \square \mathrm{CHCl}_{3}: 24.3,25.6,39.8,41.2,49.2,52.3,55.5,57.4,57.7,62.4$, 74.3, 96.9, 107.0, 124.0, 125.7, 141.0, 159.8, 175.8, 179.7; IR (NaCl/neat) 2972, 2359, 1711, 1633, $1558,1506,1457,1341,1194,1153,1020,771 \mathrm{~cm}^{-1}$; HRMS (FAB+) calcd for $\mathrm{C}_{19} \mathrm{H}_{27} \mathrm{O}_{5} \mathrm{~N}_{2}(\mathrm{~m} / \mathrm{z})$ 363.1920 , found $(\mathrm{m} / \mathrm{z}) 363.1923$.

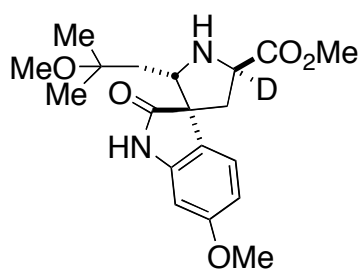

methyl ester of 10; H/D exchange

$[\square]_{D}^{25}=-17.6\left(\mathrm{CHCl}_{3}, \mathrm{c}=0.25\right) ;{ }^{1} \mathrm{H}$ NMR $\left(400 \mathrm{MHz}, \mathrm{CDCl}_{3}\right) \square \mathrm{CHCl}_{3}: 0.96(\mathrm{~d}, \mathrm{~J}=14.0 \mathrm{~Hz}, 1 \mathrm{H}), 1.05(\mathrm{~s}$, 3H), $1.13(\mathrm{~s}, 3 \mathrm{H}), 1.28(\mathrm{dd}, \mathrm{J}=8.8,14.0 \mathrm{~Hz}, 1 \mathrm{H}), 2.31$ (d, J= $12.6 \mathrm{~Hz}, 1 \mathrm{H}), 2.50(\mathrm{~d}, \mathrm{~J}=12.6 \mathrm{~Hz}, 1 \mathrm{H}$ ), $3.12(\mathrm{~s}, 3 \mathrm{H}), 3.73(\mathrm{~d}, \mathrm{~J}=8.8 \mathrm{~Hz}, 1 \mathrm{H}), 3.78(\mathrm{~s}, 3 \mathrm{H}), 3.80(\mathrm{~s}, 3 \mathrm{H}), 6.45$ (d, J=2.3 Hz, 1H), 6.55 (dd, J= 2.3, $8.2 \mathrm{~Hz}, 1 \mathrm{H}), 7.35$ (d, J= $8.2 \mathrm{~Hz}, 1 \mathrm{H}), 7.54$ (bs, $1 \mathrm{H}) ;{ }^{13} \mathrm{C} \mathrm{NMR}\left(100 \mathrm{MHz}, \mathrm{CDCl}_{3}\right) \square \mathrm{CHCl}_{3}: 24.2$, 25.6, 39.7, 41.2, 49.3, 52.4, 55.5, 57.3, 57.7, 62.4, 74.3, 97.0, 107.0, 123.9, 125.8, 140.9, 159.9, 175.7, 179.5; IR (NaCl/neat) 1710, 1630, 1505, 1461, 1270, 1244, 1193, 1153, $1122 \mathrm{~cm}^{-1}$; HRMS (FAB+) calcd for $\mathrm{C}_{19} \mathrm{H}_{25} \mathrm{DO}_{5} \mathrm{~N}_{2}(\mathrm{~m} / \mathrm{z}) 363.1904$, found $(\mathrm{m} / \mathrm{z}) 363.1906$. 


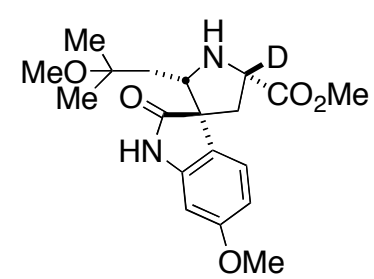

methyl ester of epi-10; H/D exchange

$[\square]_{D}^{25}=-18.6\left(\mathrm{CHCl}_{3}, \mathrm{c}=0.167\right) ;{ }^{1} \mathrm{H}$ NMR $\left(400 \mathrm{MHz}, \mathrm{CDCl}_{3}\right) \square \mathrm{CHCl}_{3}: 1.02(\mathrm{~d}, \mathrm{~J}=14.4 \mathrm{~Hz}, 1 \mathrm{H}), 1.08$ (s, 3H), 1.10 (s, 3H), 1.31 (dd, J= 9.2, $14.4 \mathrm{~Hz}, 1 \mathrm{H}), 2.13$ (d, J= $13.8 \mathrm{~Hz}, 1 \mathrm{H}), 2.76$ (d, J= $13.8 \mathrm{~Hz}$, $1 \mathrm{H}$ ), 3.09 (s, 3H), 3.54 (d, J=9.2 Hz, 1H), $3.77(\mathrm{~s}, 3 \mathrm{H}$ ), 3.79 (s, 3H), 6.44 (d, J=2.4 Hz, 1H), 6.54 (dd, $\mathrm{J}=2.4,8.0 \mathrm{~Hz}, 1 \mathrm{H}), 7.24(\mathrm{bs}, 1 \mathrm{H}), 7.41(\mathrm{~d}, \mathrm{~J}=8.0 \mathrm{~Hz}, 1 \mathrm{H}) ;{ }^{13} \mathrm{C} \mathrm{NMR}\left(100 \mathrm{MHz}, \mathrm{CDCl}_{3}\right) \square \mathrm{CHCl}_{3}: 24.3$, 25.4, 38.0, 40.6, 41.2, 49.2, 52.3, 55.5, 57.4, 65.0, 74.1, 97.0, 107.1, 123.4, 125.7, 140.9, 159.8, 175.7, 180.1; IR (NaCl/neat) 1722, 1630, 1506, 1463, 1275, 1194, 1155, 1123, $1077 \mathrm{~cm}^{-1}$; HRMS $(\mathrm{FAB}+)$ calcd for $\mathrm{C}_{19} \mathrm{H}_{25} \mathrm{DO}_{5} \mathrm{~N}_{2}(\mathrm{~m} / \mathrm{z}) 363.1904$, found $(\mathrm{m} / \mathrm{z}) 363.1910$.
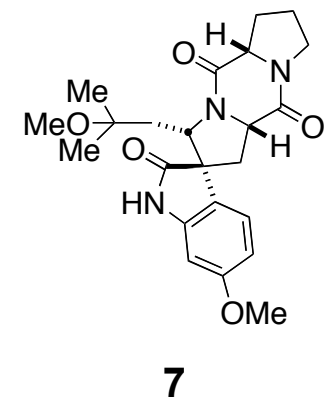

$[\square]_{D}^{25}=-60.0\left(\mathrm{CHCl}_{3}, \mathrm{c}=0.33\right) ;{ }^{1} \mathrm{H} \mathrm{NMR}\left(400 \mathrm{MHz}, \mathrm{CDCl}_{3}\right) \square \mathrm{CHCl}_{3}: 0.93(\mathrm{~s}, 6 \mathrm{H}), 1.86-2.24(\mathrm{~m}, 5 \mathrm{H})$, 2.30-2.66 (m, 3H), $2.83(\mathrm{~s}, 3 \mathrm{H}), 3.57(\mathrm{dd}, \mathrm{J}=5.4,8.2 \mathrm{~Hz}, 2 \mathrm{H}), 3.79(\mathrm{~s}, 3 \mathrm{H}), 4.20-4.27(\mathrm{~m}, 2 \mathrm{H}), 4.77$ (t, $\mathrm{J}=8.8 \mathrm{~Hz}, 1 \mathrm{H}), 6.43(\mathrm{~s}, 1 \mathrm{H}), 6.54(\mathrm{~d}, \mathrm{~J}=8.4 \mathrm{~Hz}, 1 \mathrm{H}), 7.04(\mathrm{~d}, \mathrm{~J}=8.4 \mathrm{~Hz}, 1 \mathrm{H}), 7.64(\mathrm{bs}, 1 \mathrm{H}) ;{ }^{13} \mathrm{C}$ NMR $\left(100 \mathrm{MHz}, \mathrm{CDCl}_{3}\right) \square \mathrm{CHCl}_{3}:$ 23.3, 23.6, 24.9, 27.9, 30.0, 34.9, 40.8, 45.0, 48.7, 55.5, 58.4, 59.8, 61.4, 74.0, 97.1, 106.5, 121.4, 126.3, 142.0, 160.2, 166.7, 168.1, 181.4; IR (NaCl/neat) 1716, 1665, 1633 , 1506, 1461, 1343, 1193, 1157, $732 \mathrm{~cm}^{-1}$; HRMS (FAB+) calcd for $\mathrm{C}_{23} \mathrm{H}_{30} \mathrm{O}_{5} \mathrm{~N}_{3}(\mathrm{~m} / \mathrm{z}) 428.2185$, found $(\mathrm{m} / \mathrm{z}) 428.2193$. 


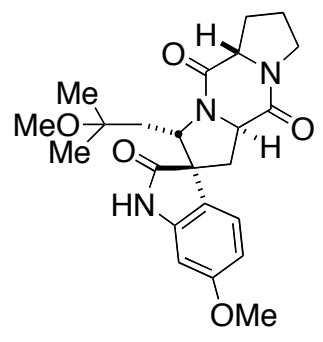

12

$[\square]_{D}^{25}=-77.0\left(\mathrm{CHCl}_{3}, \mathrm{c}=0.2\right) ;{ }^{1} \mathrm{H}$ NMR $\left(400 \mathrm{MHz}, \mathrm{CDCl}_{3}\right) \square \mathrm{CHCl}_{3}: 1.02(\mathrm{~s}, 3 \mathrm{H}), 1.19(\mathrm{~s}, 3 \mathrm{H}), 1.76-$ $1.86(\mathrm{~m}, 2 \mathrm{H}), 1.87-2.08(\mathrm{~m}, 3 \mathrm{H}), 2.41-2.53(\mathrm{~m}, 3 \mathrm{H}), 2.93(\mathrm{~s}, 3 \mathrm{H}), 3.40$ (ddd, J= 3.6, 9.4, $12.4 \mathrm{~Hz}, 1 \mathrm{H})$, $3.80(\mathrm{~s}, 3 \mathrm{H}), 3.94(\mathrm{dt}, \mathrm{J}=12.4,8.3 \mathrm{~Hz}, 1 \mathrm{H}), 4.22(\mathrm{dd}, \mathrm{J}=5.4,11.4 \mathrm{~Hz}, 1 \mathrm{H}), 4.61(\mathrm{t}, \mathrm{J}=8.8 \mathrm{~Hz}, 1 \mathrm{H})$, $4.81(\mathrm{t}, \mathrm{J}=6.8 \mathrm{~Hz}, 1 \mathrm{H}), 6.47(\mathrm{~s}, 1 \mathrm{H}), 6.54(\mathrm{~d}, \mathrm{~J}=8.2 \mathrm{~Hz}, 1 \mathrm{H}), 7.09(\mathrm{~d}, \mathrm{~J}=8.2 \mathrm{~Hz}, 1 \mathrm{H}), 7.58(\mathrm{bs}, 1 \mathrm{H})$; ${ }^{13} \mathrm{C} \mathrm{NMR}\left(100 \mathrm{MHz}, \mathrm{CDCl}_{3}\right) \square \mathrm{CHCl}_{3}: 21.7,23.2,24.7,30.0,40.6,40.7,44.7,48.5,54.8,55.5,58.8$, $60.7,61.0,74.4,97.5,106.7,121.3,125.9,142.0,160.3,163.6,165.8,181.0 ; \mathrm{IR}$ (NaCl/neat) 2927, $1718,1653,1506,1456,1343,1303,1194,1157 \mathrm{~cm}^{-1} ;$ HRMS (FAB+) calcd for $\mathrm{C}_{23} \mathrm{H}_{30} \mathrm{O}_{5} \mathrm{~N}_{3}(\mathrm{~m} / \mathrm{z})$ 428.2185, found $(\mathrm{m} / \mathrm{z}) 428.2193$.

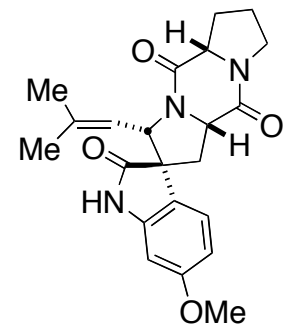

1, spirotryprostatin A

$[\square]_{\mathrm{D}}^{25}=-30.5\left(\mathrm{CHCl}_{3}, \mathrm{C}=0.2\right) ;{ }^{1} \mathrm{H} \mathrm{NMR}\left(400 \mathrm{MHz}, \mathrm{CDCl}_{3}\right) \square \mathrm{CHCl}_{3}: 1.17(\mathrm{~s}, 3 \mathrm{H}), 1.64(\mathrm{~s}, 3 \mathrm{H}), 1.86-2.08$ (m, 2H), 2.20-2.39 (m, 2H), 2.38 (dd, J= 7.2, $13.5 \mathrm{~Hz}, 1 \mathrm{H}), 2.60$ (dd, J=10.7, $13.5 \mathrm{~Hz}, 1 \mathrm{H}$ ), 3.50-3.68 (m, 2H), 3.79 (s, 3H), 4.27 (t, $8.4 \mathrm{~Hz}, 1 \mathrm{H}$ ), 4.77 (d, J=9.0 Hz, 1H), 4.99 (dd, J= 7.2, $10.7 \mathrm{~Hz}, 1 \mathrm{H}$ ), 5.02 (d, J= $9.0 \mathrm{~Hz}, 1 \mathrm{H}), 6.41(\mathrm{~d}, \mathrm{~J}=2.4 \mathrm{~Hz}, 1 \mathrm{H}), 6.49$ (dd, J= 2.4, $8.5 \mathrm{~Hz}, 1 \mathrm{H}), 6.92(\mathrm{~d}, \mathrm{~J}=8.5 \mathrm{~Hz}, 1 \mathrm{H}), 7.48$ (bs, $1 \mathrm{H}) ;{ }^{13} \mathrm{C}$ NMR $\left(100 \mathrm{MHz}, \mathrm{CDCl}_{3}\right) \square \mathrm{CHCl}_{3}: 18.0,23.7,25.5,27.4,34.4,45.2,55.5,58.5,60.2$, 60.2, 61.0, 96.6, 106.7, 118.7, 121.4, 127.3, 138.4, 141.6, 160.4, 167.1, 168.2, 180.5; IR (NaCl/neat) 2924, 1716, 1669, 1653, 1635, 1507, 1457, 1419, 1340, $1157 \mathrm{~cm}^{-1}$; HRMS (FAB+) calcd for $\mathrm{C}_{22} \mathrm{H}_{26} \mathrm{O}_{4} \mathrm{~N}_{3}(\mathrm{~m} / \mathrm{z}) 396.1923$, found $(\mathrm{m} / \mathrm{z}) 396.1909$. 


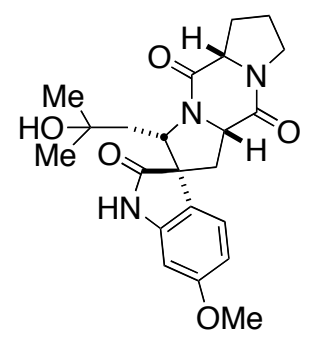

13

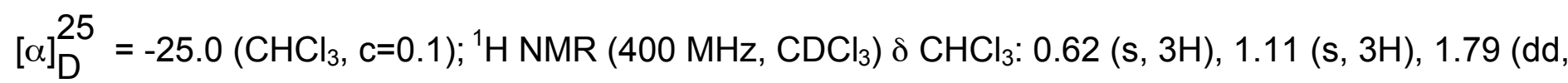
$\mathrm{J}=4.3,15.4 \mathrm{~Hz}, 1 \mathrm{H}), 1.92(\mathrm{dd}, \mathrm{J}=4.3,15.4 \mathrm{~Hz}, 1 \mathrm{H}), 1.89-2.10(\mathrm{~m}, 2 \mathrm{H}), 2.16-2.40(\mathrm{~m}, 2 \mathrm{H}), 2.46$ (dd, $\mathrm{J}=8.7,13.6 \mathrm{~Hz}, 1 \mathrm{H}), 2.65(\mathrm{dd}, \mathrm{J}=8.7,13.6 \mathrm{~Hz}, 1 \mathrm{H}), 3.60(\mathrm{dd}, \mathrm{J}=5.4,8.2 \mathrm{~Hz}, 2 \mathrm{H}), 3.79(\mathrm{~s}, 3 \mathrm{H}), 4.29$ $(\mathrm{t}, \mathrm{J}=8.2 \mathrm{~Hz}, 1 \mathrm{H}), 4.36(\mathrm{t}, \mathrm{J}=4.3 \mathrm{~Hz}, 1 \mathrm{H}), 4.45(\mathrm{bs}, 1 \mathrm{H}), 4.87(\mathrm{t}, \mathrm{J}=8.7 \mathrm{~Hz}, 1 \mathrm{H}), 6.46(\mathrm{~d}, \mathrm{~J}=2.4 \mathrm{~Hz}$, $1 \mathrm{H}), 6.56$ (dd, J=2.4, $8.4 \mathrm{~Hz}, 1 \mathrm{H}), 6.97$ (d, J= $8.4 \mathrm{~Hz}, 1 \mathrm{H}), 7.40$ (bs, $1 \mathrm{H}) ;{ }^{13} \mathrm{C} \mathrm{NMR}\left(100 \mathrm{MHz}, \mathrm{CDCl}_{3}\right)$ $\square \mathrm{CHCl}_{3}: 23.6,27.0,27.7,31.5,33.9,43.3,45.2,55.5,59.0,59.4,61.0,68.7,77.2,97.5,107.1,120.4$, 126.3, 142.0, 160.7, 168.1, 168.3, 181.4; IR (NaCl/neat) 2923, 2850, 1717, 1683, 1652, 1636, 1507, 1456, 1433, $1158 \mathrm{~cm}^{-1}$; HRMS (FAB+) calcd for $\mathrm{C}_{22} \mathrm{H}_{28} \mathrm{O}_{5} \mathrm{~N}_{3}(\mathrm{~m} / \mathrm{z}) 414.2029$, found $(\mathrm{m} / \mathrm{z}) 414.2020$.

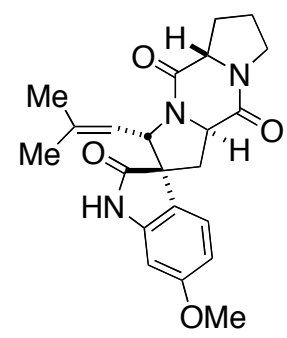

9-epi-spirotryprostatin A (14)

$[\square]_{D}^{25}=+30.0\left(\mathrm{CHCl}_{3}, \mathrm{C}=0.4\right) ;{ }^{1} \mathrm{H} \mathrm{NMR}\left(400 \mathrm{MHz}, \mathrm{CDCl}_{3}\right) \square \mathrm{CHCl}_{3}: 1.42(\mathrm{~s}, 3 \mathrm{H}), 1.67(\mathrm{~s}, 3 \mathrm{H}), 1.85-$ $2.10(\mathrm{~m}, 2 \mathrm{H}), 2.30(\mathrm{dt}, \mathrm{J}=5.5,8.2 \mathrm{~Hz}, 2 \mathrm{H}), 2.51(\mathrm{dd}, \mathrm{J}=9.0,13.4 \mathrm{~Hz}, 1 \mathrm{H}), 2.94(\mathrm{dd}, 5.2,13.4 \mathrm{~Hz}, 1 \mathrm{H}$ ), 3.57 (ddd, J= 3.5, 8.2, $11.6 \mathrm{~Hz}, 1 \mathrm{H}$ ), 3.68 (dt, J= 11.6, $8.2 \mathrm{~Hz}, 1 \mathrm{H}), 3.79(\mathrm{~s}, 3 \mathrm{H}), 4.24(\mathrm{t}, \mathrm{J}=8.2 \mathrm{~Hz}$, $1 \mathrm{H}), 4.62(\mathrm{dd}, \mathrm{J}=5.2,9.0 \mathrm{~Hz}, 1 \mathrm{H}), 5.11(\mathrm{~s}, 2 \mathrm{H}), 6.46(\mathrm{~d}, \mathrm{~J}=2.3 \mathrm{~Hz}, 1 \mathrm{H}), 6.51(\mathrm{dd}, \mathrm{J}=2.3,8.3 \mathrm{~Hz}, 1 \mathrm{H})$, 7.01 (d, J= 8.3 Hz, 1H), 7.88 (bs, $1 \mathrm{H}) ;{ }^{13} \mathrm{C}$ NMR (100 MHz, $\left.\mathrm{CDCl}_{3}\right) \square \mathrm{CHCl}_{3}: 18.1,23.3,25.7,27.8$, $35.9,45.7,54.2,55.5,58.9,60.0,61.6,97.0,106.9,119.1,119.6,125.7,138.4,142.1,160.3,165.6$, 166.1, 179.7; IR (NaCl/neat) 1720, 1662, 1598, 1506, 1426, 1342, 1310, 1278, 1193, $1156 \mathrm{~cm}^{-1}$; HRMS (FAB+) calcd for $\mathrm{C}_{22} \mathrm{H}_{26} \mathrm{O}_{4} \mathrm{~N}_{3}(\mathrm{~m} / \mathrm{z}) 396.1923$, found $(\mathrm{m} / \mathrm{z}) 396.1915$. 